\title{
Aportaciones de expertos del ámbito académico y diseñadores para la creación de experiencias de juego cívicas
}

\author{
Jorge Oceja ${ }^{1}$ y Natalia González-Fernández ${ }^{2}$ \\ ${ }^{1}$ Universidad de Cantabria y Universidad Internacional de Valencia -España \\ ${ }^{2}$ Universidad de Cantabria - España
}

\section{RESUMEN}

El propósito de esta investigación es obtener orientaciones para el diseño de experiencias de juego que puedan promover la competencia cívica. Para ello se ha trabajado con dos colectivos: expertos del ámbito académico que desarrollan su labor en gamelabs europeos y diseñadores de exitosos juegos comerciales de carácter cívico. En particular trabajamos con miembros del Center for Applied Game Research (CEAGAR) de la Universidad de Aalborg en Copenhague y con diseñadores de Bulgaria, Polonia y Estados Unidos. El estudio se centra en los siguientes aspectos: elementos que han de ser incluidos en estas experiencias, consideraciones del proceso de diseño e importancia de las formas de juego emergentes Los dos colectivos coinciden en varios aspectos (necesidad de ofrecer a los jugadores acciones y emociones significativas, la importancia de una retroalimentación equilibrada o la influencia que tendrán los juegos cross-media en el futuro) pero a su vez aportan visiones propias; por ejemplo, los diseñadores subrayan la importancia de la transgresión y la superación de tabúes y los expertos académicos la necesidad de otorgar más importancia a las ideas que a los aspectos tecnológicos.

Palabras clave: videojuegos, gamificación, competencia cívica, juegos cívicos, diseño de juegos.

\section{Contributions from Academic Experts and Game Designers to Develop Civic Game experiences}

\section{ABSTRACT}

This research seeks to generate practical guidelines based on the contributions of experts in the academic field and game designers to create game experiences that can promote civic competence. We have worked with two groups: academic experts from the Center for Applied Game Research (CEAGAR) at Aalborg University in Copenhagen and game designers from Bulgaria, Poland and United States. The study focuses on what game elements should be included in these experiences, other considerations in the design process and the importance of new game forms. Both groups point out common aspects (need of offering the player meaningful actions and emotions, the importance of a balanced feedback and the increasing importance of cross-media games) but they also share their own visions; for instance, designers highlight the need of transgression by overcoming taboos while academic experts mention the importance of prioritizing ideas over technology.

Keywords: video games, gamification, civic competence, civic games, game design. 


\section{Introducción}

La actividad de jugar y los juegos en sí mismos son una manifestación esencial del desarrollo del ser humano (Caillois, 1961; Huizinga, 1949) y nuestra época un momento particularmente permeable a la utilización de las metáforas, valores y elementos presentes en los mismos. Tal y como indica Raessens (2006) vivimos inmersos en una ludificación de la cultura.

Diversos autores han reflexionado sobre las posibilidades que en este contexto ofrecen las experiencias de juego para la promoción de la competencia cívica (Ashtari \& de Lange, 2019; Neys \& Jansz, 2019), especialmente si son utilizadas desde una perspectiva crítica (Marín, 2006). Para entender mejor estas cuestiones repasaremos la consolidación del término competencia cívi$c a$ en el marco europeo y, posteriormente, revisaremos los distintos productos jugables que han permitido abordarla.

\subsection{La competencia cívica en el marco de la Unión Europea}

La consolidación en Europa del enfoque competencial está relacionada con distintos eventos y momentos históricos (Álvarez-Arregui, E., 2019). El primero de ellos es el plan de la Unión Europea denominado Estrategia de Lisboa (Consejo de Europa, 2000), que pretendía convertir la economía de la UE en la más competitiva del mundo para 2010, basándose en el conocimiento, el empleo y (aunque incluido posteriormente), en el desarrollo sostenible. Estas orientaciones tomaron forma en la estrategia de Educación y Formación 2010 y se desarrollaron posteriormente en los planes Europa 2020 y Educación y Formación 2020 (Consejo Europeo, 2010). Todos ellos contribuyeron a sentar las bases del modelo competencial en las políticas europeas. En este contexto, el Consejo Europeo (2001) adoptó el Informe de la Comisión Europea titulado Futuros objetivos precisos de los sistemas educativos y que incluía en su punto 1.2 la necesidad de promover la adquisición de las competencias necesarias para poder abordar una sociedad basada en el conocimiento.

A partir de ese momento, todos los territorio europeos asumen este enfoque y el Parlamento y el Consejo recogen en 2006 el informe de la Comisión, Marco europeo de referencia para las competencias clave del aprendizaje permanente y que se resume en un documento más divulgativo publicado el año siguiente (Comisión Europea, 2007). En dichos documentos se establecen las ocho competencias clave que todos conocemos: Comunicación en la lengua materna, comunicación en lenguas extranjeras, competencia matemática y competencias básicas en Ciencia y Tecnología, competencia digital, competencia de aprender a aprender, competencia cívica y social, sentido de la iniciativa y espíritu de empresa y conciencia y expresiones culturales.

Estos documentos se refieren a la competencia cívica y social de la siguiente manera:

Estas competencias incluyen las personales, interpersonales e interculturales y recogen todas las formas de comportamiento que preparan a las personas para participar de una manera eficaz y constructiva en la vida social y profesional, especialmente en sociedades cada vez más diversificadas, $\mathrm{y}$, en su caso, para resolver conflictos (p.9)

No obstante, la referencia explícita a la competencia cívica viene a continuación cuando se afirma que:

La competencia cívica prepara a las personas para participar plenamente en la vida cívica gracias al conocimiento de conceptos y estructuras sociales y políticas, y al compromiso de participación activa y democrática. (p. 9)

Una exploración más detallada de los citados documentos nos permite listar todos los conocimientos, capacidades y actitudes concretas en los que se manifestaría dicha competencia.

\subsection{Clasificación de experiencias de juego para la promoción de la competencia cívica}

En los últimos años distintos agentes (estudios de videojuegos, organizaciones no gubernamentales, administración pública, universidades, etc.) han desarrollado experiencias para abordar de forma más o menos explícita los indicadores que acabamos de mostrar. Sin embargo, son pocos los intentos por comprender y clasificar estas propuestas. Tras la revisión de distintos modelos, Oceja y González-Fernández (2017) proponen partir del término Experiencia de juego y, posteriormente, utilizar la clásica diferenciación de Deterding entre productos que usan elementos aislados de los juegos (gamificación) y aquellos que pueden considerarse juegos "completos" (juegos) (Deterding, Dixon, et al., 2011). Dentro de la gamificación cívica (donde nos encontraríamos con apps vinculadas a temas como la ciudad inteligente, la acción política o el consumo responsable) no se establecerían más distinciones, pero dentro de los juegos cívicos, se recupera el concepto clásico de círculo mágico (Huizinga, 1949) para distinguir ente juegos ubicuos (Montola et al., 2009) y no ubicuos.

Cuando analizamos estos productos más convencionales, distinguimos a su vez entre juegos diseñados para contextos formales (juegos educativos [Egenfeldt-Nielsen, 2005; Facer et al., 2003]), juegos comerciales que pueden ser utilizados tanto en contextos formales como informales a los que la literatura se refiere como Commercial Off-the-Shelf Games (COTS) y los juegos diseñados para ser jugados en contextos informales (juegos persuasivos). Dentro de esta última categoría, se diferenciaría entre dos productos: los juegos diseñados para informar o crear una opinión sobre un asunto concreto (por lo tanto, distribuidos a través de medios de comunicación) y los creados por pequeños estudios o desarrolladores independientes y distribuidos de una forma más convencional.

No obstante, como indican diversos autores (Bogost, 2015; Fuchs, Fizek, \& Ruffino, 2014), la mayoría de estas experiencias, en particular los videojuegos educativos y la gamificación cívica, no han sido satisfactorias (Oceja y González-Fernández, 2018).

Por todo ello, el propósito de esta investigación es obtener orientaciones prácticas basadas en las aportaciones de expertos del ámbito académico y diseñadores de referencia para crear experiencias de juego que pudieran promover la competencia cívica. Para ello se trabajó con investigadores del Center for Applied Game Research (CEAGAR) de la Universidad de Aalborg en Copenhague y con diseñadores internacionales de videojuegos cuyos productos han atendido la dimensión cívica. El trabajo ha servido para dar respuesta a las siguientes preguntas de investigación: ¿Qué recomendaciones ofrece cada uno de estos colectivos para el diseño de experiencias que pudieran promover la competencia cívica? ¿En qué medida las orientaciones de diseño son redundantes o exclusivas de cada uno de los grupos? 


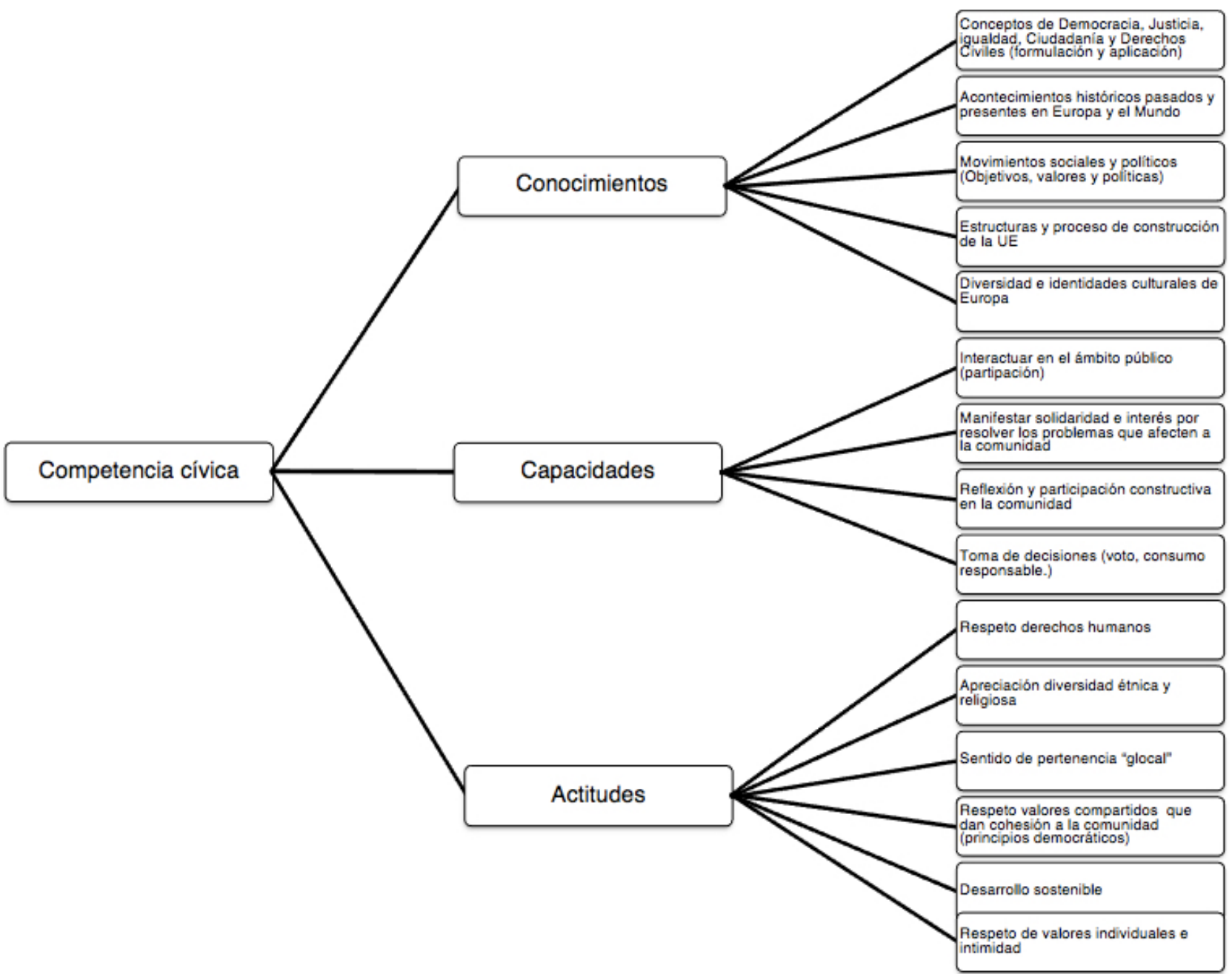

Figura 1. Conocimientos, capacidades y actitudes de la competencia cívica. Elaboración propia a partir de la Decisión del Parlamento Parlamento Europeo y del Consejo. Diario Oficial de la Unión Europea, de 24 de noviembre de 2006

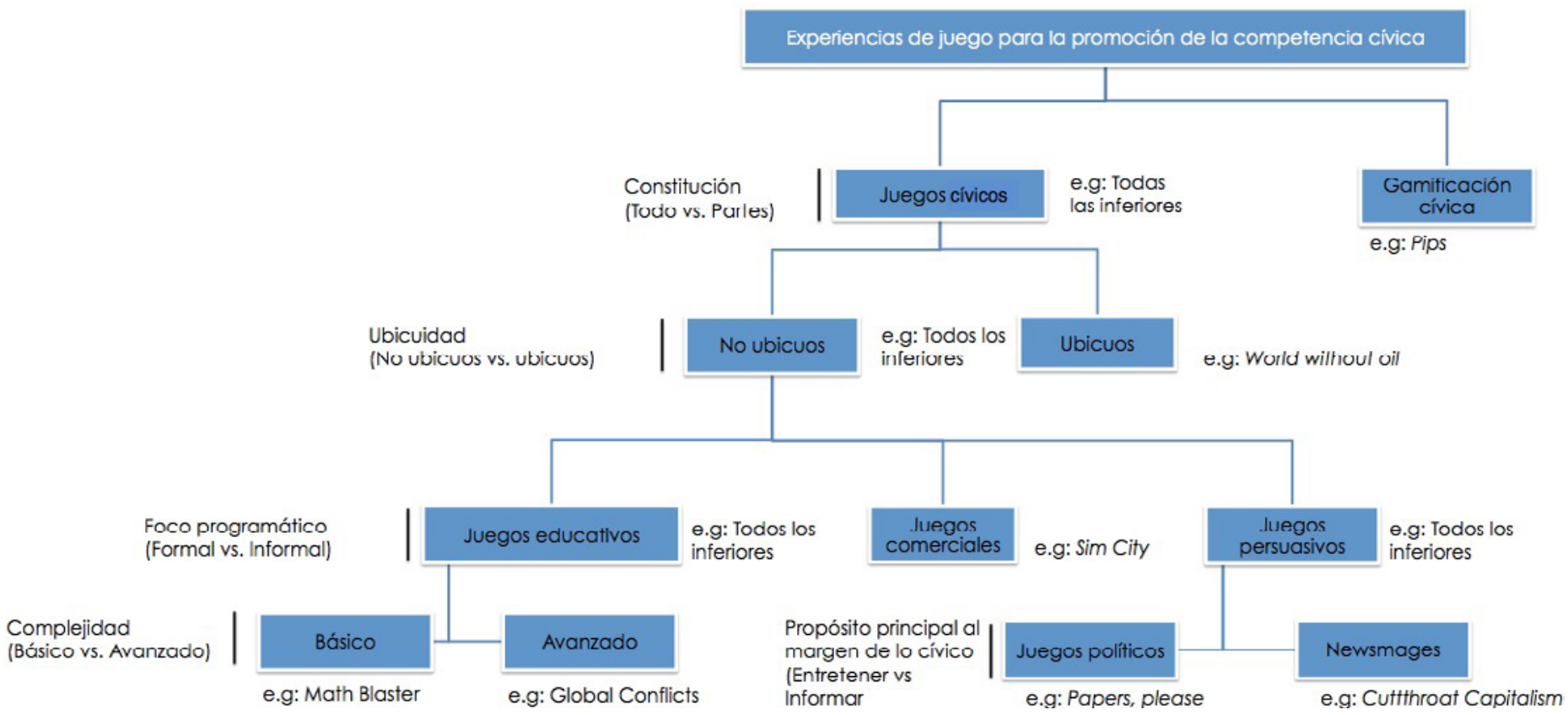

Figura 2. Clasificación de las Experiencias de juego para la promoción de la competencia cívica 


\section{Metodología}

La investigación se basó en un acercamiento cualitativo que permitió obtener información del grupo de expertos a través de un focus group y de los diseñadores a través de distintas entrevistas administradas vía email. En las próximas líneas detallamos cómo fue el proceso de selección de la muestra, la construcción de los instrumentos y la ejecución del procedimiento metodológico.

\subsection{Muestra}

Tal y como indican distintos autores (Creswell, 2011; Mertens, 2014), en los acercamientos cualitativos el objetivo principal no es generalizar los resultados a una población, sino explorar en profundidad un determinado fenómeno, realizando una selección intencionada de los individuos y espacios que se consideren más relevantes.

Para seleccionar al grupo de expertos buscábamos un espacio universitario de referencia internacional en nuestro ámbito de estudio. Los criterios de selección fueron el estar formado por un equipo interdisciplinar (con profesionales, al menos, de la educación, la comunicación y el diseño de juegos), que combinara la investigación con la generación de productos a través de labs o espacios similares y que mantuviera contacto con otros agentes del sector como escuelas, administración pública y diseñadores de videojuegos. Tras analizar distintas opciones, el centro seleccionado fue el CEAGAR de la Universidad de Aalborg en Copenhague al cumplir, de forma explícita, todas estas características. Para el focus group fueron convocados sus dos codirectores (Henrik Schønau Fog y Thorkild Hanghøj y dos de sus miembros Stine Ejsing-Duun y Rikke Magnusen). El proceso, por tanto, fue propositivo y se acercó a lo que algunos autores denominan elección de un caso instrumental o típico (Hartas, 2015; Martínez-Salgado, 2012) en el que el grupo en cuestión es considerado particularmente relevante y representativo del objeto de estudio.

Por otro lado, acceder a una población como la de diseñadores de juegos comerciales de carácter cívico no es una tarea sencilla. Los estudios demuestran que los productos cívicos no son los más jugados y que muchas veces se circunscriben a la escena independiente. Por ejemplo, Oceja y González-Fernández (2020), trabajando con 610 estudiantes universitarios, comprobaron que solo 10 de sus juegos favoritos podían considerarse susceptibles de promover la competencia cívica. (Tabla1).

Para seleccionar nuestra muestra partimos de estos juegos y establecimos como criterio el ser el diseñador principal o tener un papel relevante en el diseño de alguno de ellos.

Tras múltiples intentos, que se extendieron a lo largo de varios meses a través de correo electrónico, llamadas y redes sociales, se obtuvo la colaboración de Alan Gershenfeld (Diseñador de Never Alone y cofundador de Eline Media), Pawel Miechowski (Codiseñador y guionista de This War of Mine en 11 Bit Studios) y Bisser Dyankov (Codiseñador de la serie Trópico en Haenimont Games).

\subsection{Instrumentos}

Se diseñaron y validaron dos instrumentos para la recogida de datos: el guion para la realización de un focus group con los expertos y el guion de la entrevista en la que participarían los diseñadores. Aunque con peculiaridades al tratarse de técnicas de recogida de datos diferentes, ambos instrumentos presentaron elementos comunes.
Tabla 1

Juegos seleccionados por los estudiantes que pueden promover la competencia cívica agrupados por géneros

\begin{tabular}{lll}
\hline Género & Juego & Compañías \\
\hline Simulación & SimCity & $\begin{array}{l}\text { Will Bright y Maxis (Eletro- } \\
\text { nic Arts) }\end{array}$ \\
& $\begin{array}{l}\text { Geo Political } \\
\text { Simulator }\end{array}$ & Eversim \\
\hline Estrategia & Euro of Empires & Ensamble Studios \\
& Salis & Actualmente Microsoft) \\
& RUSE & $\begin{array}{l}\text { Paradox Development Stu- } \\
\text { dio }\end{array}$ \\
& Trópico & Eugen Systems \\
& Haemimont Games y \\
\hline Aventura & This war of mine & 11 bit Studios \\
\hline Rol & Never Alone & Eline Media \\
\hline & Wakfu & $\begin{array}{l}\text { Ankama Games y Square } \\
\text { Enix }\end{array}$ \\
\hline
\end{tabular}

\subsubsection{Guion para el focus group con expertos académicos}

Las preguntas no fueron diseñadas para realizarse de forma automática, sino que constituían una guía que sería adaptada en función de la actividad de los participantes. El instrumento se validó a través de un juicio de expertos. Para la selección de los mismos se establecieron los siguientes criterios: amplia experiencia profesional vinculada a tendencias emergentes en educación y experiencias de juego o ser un experto en dichas cuestiones o en métodos de investigación. (Tabla 2)

10 de los 11 expertos completaron un cuestionario centrado en la presentación, las preguntas y la percepción general del instrumento. La valoración de la presentación fue positiva calificándose de forma favorable su claridad (50\% buena y $50 \%$ excelente), la extensión ( $40 \%$ buena y $60 \%$ excelente) y, aunque con una respuesta discordante, la adecuación a los participantes $(60 \%$ excelente, $30 \%$ buena y $10 \%$ regular). Atendimos la sugerencia de incluir una breve explicación de lo que es un focus group y comprobamos que la terminología fuera consistente en todo el instrumento.

Las preguntas también recibieron la aprobación de los expertos; consideraron adecuada su calidad (el 50\% la considera buena y el $50 \%$ excelente) y el número y orden de las mismas ( $30 \%$ bueno y un $70 \%$ excelent)e.

La percepción general del instrumento fue muy favorable valorándose de forma muy positiva su estructura (excelente para el $60 \%$ y buena para el $40 \%$ ), la adecuación a los participantes (excelente para el 60\%, buena para el 30\% y regular para el $10 \%$ ) y su extensión (excelente para el $70 \%$ y buena para el $30 \%)$. 
Tabla 2

Estructura del guion para el focus group Expertos Gamelabs Europa (EGE)

- Contextualización del estudio

Presentación

- Revisión de la estructura del focus group

- Solicitud de autorizaciones para la grabación

- A la hora de crear experiencias de juego para la promoción de la competencia cívica ¿qué elementos (ej. convenciones iconográficas, componentes más o menos análogos al mundo real, acciones que los jugadores pueden realizar o emociones que pueden experimentar) creen que ofrecen más posibilidades para motivar a los usuarios? ¿Por qué?

- ¿Qué otros aspectos vinculados con el diseño de juegos (ej. reglas, apartado gráfico, desarrollo de Diseño de expe- inteligencia artificial, etc.) creen que son imporriencias de juego tantes? ¿Por qué?

- Dado que la barrera entre gamificación y juegos en un sentido estricto es cada vez más difusa ¿cómo creen que puede influir la aparición de nuevas formas de juegos (ej. juegos ubicuos (a nivel espacial, social o temporal), juegos cross-media o juegos basados en nuevas formas de interacción (audio-based games, juegos de compañías como Simogo, etc.) en la generación de experiencias que promuevan esta competencia?

Otras cuestiones • ¿Desea añadir alguna otra cuestión?

\subsubsection{Guion de las entrevistas con diseñadores}

Para recoger información de los diseñadores, dada su ubicación en tres países diferentes, preparamos una entrevista para ser administrada vía email con preguntas abiertas.

Tabla 3

Estructura de la entrevista Diseñadores de Videojuegos (DV)

\begin{tabular}{ll}
\hline & - Contextualización del estudio \\
Presentación & - Revisión de la estructura del focus group \\
& - Solicitud de autorizaciones para la grabación \\
\hline
\end{tabular}

- ¿Por qué cree que su juego ha tenido tal trascendencia? Piense en cuestiones como cuál fue su origen, el proceso, cómo se diseño, las

Bloque 2. razones por las que cree que los jugadores le

Diseño de experiencias de juego otorgan esa importancia. etc.

- Dado que la barrera entre las distintas experiencias de juego es cada vez más difusa ¿cómo cree usted que puede influir la aparición de nuevas formas de juego en la generación de experiencias que promuevan la competencia cívica?

Otras cuestiones
Se realizó otro juicio de expertos siguiendo los mismos criterios profesionales y académicos que en el caso anterior. En esta ocasión el cuestionario fue respondido por 6 de los 12 jueces. La valoración de la presentación fue muy positiva, tanto su claridad (33.3\% buena y $66.7 \%$ excelente) como la extensión y la adecuación a los participantes ( $16.7 \%$ buena y $83.3 \%$ excelente). Siguiendo sus indicaciones revisamos la consistencia de los términos, asegurándonos que utilizábamos el concepto Experiencia de juego para cualquier experiencia jugable.

La calidad de las preguntas volvió a ser muy bien valorada (16.7\% buena y $83.3 \%$ excelente) y todos los expertos consideraron que el orden y el número de preguntas era adecuado. La percepción general del instrumento fue muy favorable, valorándose de forma muy positiva tanto la estructura y adecuación a los participantes $(16,7 \%$ buena y $83.3 \%$ excelente) como su extensión (33.3\% buena y $66.7 \%$ excelente).

Con ambos instrumentos se siguió el criterio de verificación de los informantes (George \& Apter, 2004), permitiendo en todo momento que éstos accedieran y modificaran, tanto la información suministrada, como la interpretación que se hizo de la misma durante el desarrollo de la investigación.

\subsection{Procedimiento}

Para dar respuesta a las preguntas, analizamos de forma independiente el contenido del focus group con los expertos y las entrevistas con los diseñadores. El procedimiento fue ligeramente distinto ya que al completar los investigadores las entrevistas a través del correo electrónico se evitó el proceso de transcripción. En el caso del focus group, tuvimos que realizar de forma manual la transcripción desde el programa iTunes para generar los archivos .doc finales.

Todos los documentos fueron trasladados al software para tratamiento cualitativo de datos ATLAS.ti versión 1.6.0 para Mac. En ambos casos (entrevistas y focus group), comenzamos con una codificación en busca de conceptos relevantes (Bogdan \& Biklen, 2007; Tesch, 2013). Posteriormente agrupamos los códigos para evitar solapamientos y los colapsamos en categorías. Finalmente generamos dos diagramas en forma de árbol jerárquico para representar tanto las categorías existentes como el sistema de relaciones entre las mismas.

El proceso tuvo un carácter principalmente inductivo con aportaciones y categorías que emergieron de los propios datos en la línea de los análisis propuestos por la inducción analítica (Robinson, 2000) o la teoría fundamentada (Strauss \& Corbin, 1997). Aunque no sistematizamos el procedimiento con todos los protocolos que sugiere dicha teoría, sí que atendimos a alguna de sus características principales. Por ejemplo, seguimos las recomendaciones de Schettini (2015) y combinamos una codificación abierta en la que los datos "se abren para sacar a la luz los pensamientos, las ideas y los significados" (p. 37), con una codificación axial posterior en la que establecimos las mencionadas relaciones jerárquicas entre las categorías. Asimismo, la construcción de categorías se vio influida por el método de comparación constante (Strauss \& Corbin, 1997) según el cual es necesario comparar los incidentes que van apareciendo en los datos con otros incidentes, estos con las categorías y, a su vez, las categorías con otras categorías.

Una vez construidos los árboles de cada grupo, cruzamos los datos y, siguiendo las recomendaciones de distintos autores (Miles, Huberman, \& Saldana, 2014; Spradley, 2016) construimos una tabla que integrara las categorías generadas por ambos colectivos para comprobar en qué casos coincidían y cuáles eran exclusivas de alguno de ellos. 


\section{Resultados}

Aunque ambos colectivos (expertos académicos y diseñadores) coincidieron en muchas de sus propuestas, también generaron aportaciones propias. Comenzamos mostrando las tablas resultantes del análisis de cada colectivo y revisando, a continuación, algunas de las citas que dan lugar a dichas representaciones. En la parte final mostramos una tabla resumen con las categorías compartidas y exclusivas de cada grupo. La codificación $E$ $(E 1, E 2$ y E3) corresponde a las citas de los expertos y la de D (D1, D2 y D3) a los diseñadores. (Figura 6 y 7).

Tras confirmar ambos grupos la importancia de ofrecer al jugador la posibilidad de ejecutar acciones significativas (E2: Lo importante aquí es ejecutar acciones: tienes que poner tus acciones ahí fuera, en el mundo), al abordar las emociones que han de atenderse en el proceso de diseño, los investigadores añadieron a las ideas de camaradería y desafío, apuntadas por los diseñadores (D1: Los juegos que no terminan y generan comunidades robustas tienen mayores posibilidades de impacto; E2: El desafío es tan importante que sin él, ¿por qué hacer algo?), la de descubrimiento (E3: Podemos usar eventos que ocurren en la ciudad para fomentar el deseo de exploración). Más allá de los elementos a incluir, ambos grupos realizaron consideraciones sobre el proceso de diseño; a ideas compartidas como la importancia de ofrecer una retroalimentación equilibrada (D1: El jugador ha de poder repetir acciones a partir de un feedback abundante del juego) y la necesidad de adaptarse a los diferentes contextos (D1: Diseñar juegos requiere una profunda comprensión del contexto donde se van a implementar), los expertos añadieron la necesidad de simplificar algunos procesos (E2: Tengo estudiantes de artes haciendo gamificación o juegos para el cambio y lo más interesante es entender cómo pueden simplificarse los procesos) y considerar la relación entre los proyectos y la tecnología (E2: Suelo empezar con el desafío y después pensar en cómo puedo hacerlo [...] aunque alguna vez veo una tecnología y digo "wow", hay un problema que podría resolver utilizando esto). Por su parte, los diseñadores mencionaron la importancia de potenciar la autonomía de los jugadores (D1: Es necesario crear un equilibrio que mueva el interés del jugador para que pueda avanzar a su propio ritmo) $\mathrm{y}$, sobre todo, el hecho de que los juegos son una forma de arte (D2: Los juegos son una forma de arte y mientras ese arte conmueva a los jugadores, lo probarán. Provocamos la reflexión sobre distintos aspectos de la vida de la misma manera que puede buscarlo quien crea una película). Ambos colectivos atribuyeron gran importancia a la aparición de nuevas formas de juego como los juegos ubicuos (D1: Creo que hay un gran potencial para los juegos de rol en vivo para añadir energía a programas cívicos que ya existen) o las experiencias transmedia (D2: Ampliar o derribar las fronteras de los medios sólo puede ayudar). De forma específica, los expertos se refirieron a las opciones que ofrece la monitorización de comportamientos (E2: La otra cuestión es que la pervasividad de los dispositivos permite monitorizar a las personas) y los diseñadores al papel que puede jugar la realidad virtual (D3: Imagino que alguna vez tendremos algo tipo "Artillería para Wall Street", en la que simularemos pelearnos virtualmente con la policía). Finalmente, los diseñadores añadieron la necesidad de superar tabús a la hora de escoger los temas de las experiencias jugables. A partir de sus propios juegos, nos mostraron cómo pueden crearse productos basados en el humor (D3: La gente recuerda Trópico por su humor, lo que permitía jugar con muchos tabús y muchas cuestiones delicadas que serían imposibles de abordar de una forma más seria), las relaciones intergeneracionales y la transmisión oral (D1: Never Alone ex-

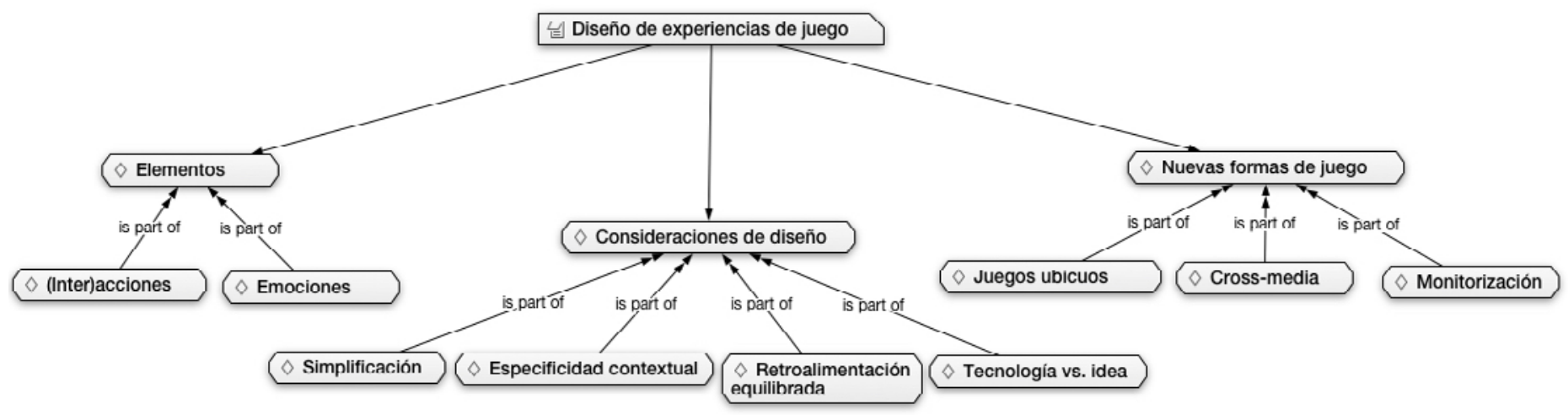

Figura 6. Red semántica Diseño de experiencias de juego, con la información obtenida de los miembros del CEAGAR

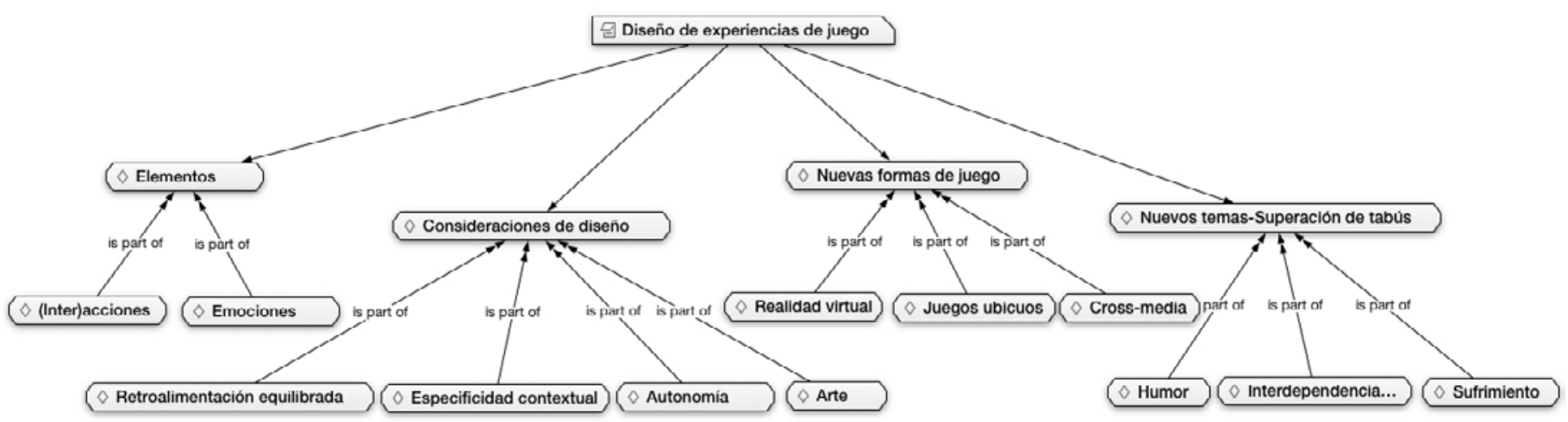

Figura 7. Red semántica Diseño de experiencias de juego con la información obtenida de los diseñadores 
plora por qué ciertas historias se han trasmitido durante miles de años intentando llevar esto al nuevo medio que son los juegos) o la exposición al sufrimiento humano (D2: Nuestro juego en cierta medida rompió el tabú de no mostrar el sufrimiento de la población civil). Estas aportaciones y el propio This War of Mine pusieron de manifiesto como un juego, aún teniendo la guerra como temática, puede aportar beneficios de carácter cívico.

A continuación, mostramos una tabla resumen que combina las categorías resultantes del tratamiento de los datos de los dos grupos. Aunque algunas categorías (habitualmente del primer nivel) tienen cierto sentido apriorístico, prácticamente todos los elementos del segundo y tercer nivel emergieron a posteriori, de forma inductiva. En verde aparecen las categorías que comparten los dos grupos, en azul las categorías exclusivas de los expertos académicos y en naranja las de los diseñadores.

\begin{tabular}{|c|c|c|}
\hline Categoría nivel 1 & Categoría nivel 2 & Categoría nivel 3 \\
\hline \multirow{4}{*}{$\begin{array}{l}\text { Elementos } \\
\text { importantes }\end{array}$} & (Inter)acciones & \\
\hline & \multirow{3}{*}{ Emociones } & Camaradería \\
\hline & & Desafío \\
\hline & & Descubrimiento \\
\hline \multirow{6}{*}{$\begin{array}{c}\text { Otras } \\
\text { consideraciones } \\
\text { de diseño }\end{array}$} & $\begin{array}{l}\text { Retroalimentación } \\
\text { equilibrada }\end{array}$ & \\
\hline & $\begin{array}{l}\text { Especificidad } \\
\text { contextual }\end{array}$ & \\
\hline & Simplificación & \\
\hline & Tecnología vs. Idea & \\
\hline & Autonomía & \\
\hline & Arte & \\
\hline \multirow{4}{*}{$\begin{array}{l}\text { Importancia de las } \\
\text { nuevas formas de } \\
\text { juego }\end{array}$} & Juegos ubicuos & \\
\hline & Cross-media & \\
\hline & Monitorización & \\
\hline & Realidad virtual & \\
\hline \multirow{3}{*}{$\begin{array}{c}\text { Importancia de } \\
\text { abordar nuevos temas }\end{array}$} & \multirow{3}{*}{$\begin{array}{l}\text { Superación } \\
\text { de tabús }\end{array}$} & Humor \\
\hline & & $\begin{array}{l}\text { Interdependencia } \\
\text { generacional }\end{array}$ \\
\hline & & Sufrimiento \\
\hline
\end{tabular}

Figura 8. Categorías compartidas y exclusivas de cada grupo (diseñadores y expertos académicos)

\section{Discusión y conclusiones}

Ambos colectivos otorgan una gran importancia a la posibilidad de ejecutar acciones y experimentar emociones significativas durante el juego. Estos datos coinciden con aportaciones que han subrayado la importancia de la dimensión emocional (Hunicke et al., 2004; Lazzaro, 2004; Vorderer et al., 2004), la necesidad de proveer al jugador de acciones significativas (Manninen, 2003; Zaffiro et al., 2015) y, sobre todo, los problemas de basar las experiencias exclusivamente en puntos, rankings y condecoraciones (Bogost, 2015; Fuchs et al., 2014). Se trata de una cuestión importante ya que gran parte de las experiencias de juego cívicas desarrolladas desde distintos ámbitos (ONGs, administración pública, etc.) y con distintos objetivos (promoción de hábitos saludables, consumo responsable, etc.) bajo el paraguas de la gamificación, suelen limitarse a una utilización básica y simplista de dichas convenciones (rankings, condecoraciones, puntos, etc.), sin entender la complejidad latente en los juegos. Este desequilibro entre la riqueza del medio y la simplicidad de muchas prácticas gamificadas, creemos que es un aspecto importante.

Dentro de la dimensión emocional se otorga una gran importancia al descubrimiento y, sobre todo, al desafío, datos coincidentes con estudios previos (Hamari et al., 2016; Lazzaro, 2004; Zagalo, Carvalho, \& Araújo, 2016). Otros autores (Fuster, 2017), reconocen la importancia de conceptos cercanos como la exploración y el logro. Esto indicaría que cuando los jugadores participan en una experiencia, quieren explorar e investigar; quieren descubrir por sí mismos el territorio que el sistema representacional del juego les ofrece, lo que debería llevarnos a crear espacios que les permitan descubrir nuevas realidades. Ambos colectivos subrayaron la importancia de la camaradería, cuestión desatendida por muchos juegos cívicos en la actualidad al omitir la dimensión social y la participación en comunidades. En la línea apuntada por diversos autores (Del Moral \& Villalustre, 2006), para promover la competencia cívica hemos de fomentar la relación entre jugadores ofreciéndoles la posibilidad de ejecutar distintas acciones, fomentando su autonomía y permitiéndoles experimentar distintas emociones.

Ambos grupos coincidieron en la importancia de ofrecer una retroalimentación equilibrada y en la conveniencia de adaptar las experiencias a los contextos de los sujetos (Brookes \& Moseley, 2012). Esto implica que, si queremos promover la capacidad de participación en el ámbito público, debemos proponer acciones reales y sobre todo viables, que los jugadores puedan ejecutar en su día a día.

Los diseñadores, además, subrayaron, tal y como apuntan varios autores (Jenkins, 2005; Schell, 2014), la importancia de atender a la dimensión artística en el proceso de diseño; es necesario emocionar a los jugadores de una manera similar a la que ocurre con otras artes plásticas y visuales.

Los dos grupos coincidieron en resaltar el papel que tendrán las formas emergentes de juego (Oceja \& González-Fernández, 2016), en particular los juegos ubicuos y las experiencias cross-media que combinen lenguajes y medios de distinto tipo, no exclusivamente digitales. Los expertos mencionaron, además, el papel relevante que tendrá la monitorización de comportamientos (cuestión que habrá de ir acompañada de consideraciones en torno a la privacidad y tratamiento de esos datos) y los diseñadores la importancia de la realidad virtual. El auge de las Escape Rooms en ciudades de todo el mundo, el éxito comercial de experiencias geolocalizadas como Ingress o Pokemon Go, o la aparición de productos híbridos, a medio camino entre distintos medios, como Device 6 de la compañía Simogo, parecen confirmar la importancia de estas nuevas formas de juego. Finalmente, los diseñadores aportaron un elemento que nos parece clave: la necesidad de superar tabús en torno a los temas y tonos que pueden guiar el diseño de experiencias de juego. En el difícil equilibrio entre convencionalidad y creatividad apuntado por Tschang (2007), creemos que el éxito de los productos que ellos representan (This war of mine y su visión del sufrimiento civil, Trópico y la mirada satírica de los sistemas dictatoriales o Never Alone y su reivindicación de la tradición oral y la interdependencia generacional) son un ejemplo de cómo miradas innovadoras pueden ser viables incluso en contextos comerciales. 
Las limitaciones más evidentes de este trabajo tienen que ver con las muestras utilizadas. Aunque desde un primer momento se optó por el trabajo con expertos del ámbito académico y diseñadores de videojuegos, la selección de estos colectivos iba a tener, forzosamente, condicionantes.

A pesar de establecer criterios para la selección de expertos, sus aportaciones no pueden generalizarse sin una reflexión previa a todos los gamelabs europeos $\mathrm{y}$, mucho menos, a todos los expertos del ámbito académico. Por ello, los autores de la presente investigación se encuentran recogiendo datos de otros espacios como el EngageLab de la Universidade do Minho en Güimaraes (Portugal) y el Gamification Lab de la Universidad Leuphana en Lunebürg (Alemania).

Por otro lado, conocer y dar voz a un colectivo como los diseñadores de videojuegos que trabajan en productos comerciales pero con un carácter cívico, es una tarea compleja. Aunque escogimos a profesionales que nos proporcionaron información relevante, con adecuados niveles de saturación y que aportaba diversidad geográfica y cultural (Bulgaria, Polonia y Estados Unidos), se trata de tres voces concretas. Sería pertinente trabajar con muestras mayores e incluir, por ejemplo, la opinión de las diseñadoras. Como dijo Bisser Dyankov con cierta ironía, "no sé si soy representativo del diseñador medio, en el caso de que ese animal exista".

Es necesario entender que, aunque los investigadores y los propios informantes hablaron frecuentemente de elementos de los juegos, el diseño es un proceso creativo y artístico cuya complejidad va más allá de la inclusión mecánica de unos u otros elementos.

Las experiencias de juego, en sus distintas manifestaciones (videojuegos, gamificación, formas de juego emergentes, etc.) son productos culturales cuyas posibilidades para promover la competencia cívica son cada vez más reconocidas. Sin embargo, su estudio en contextos académicos es relativamente novedoso y han pasado pocos años desde los primeros trabajos centrados en el aprendizaje a través de experiencias de juego (Gee, 2014; Shaffer, Squire, Halverson, \& Gee, 2005). A pesar de su juventud, la diversidad de acercamientos (psicología, filosofía, artes, ingeniería, sociología, etc.) y la enorme producción científica de los últimos años, son una muestra de la trascendencia y energía creativa de este campo de estudio. Esperamos que la mirada educativa aquí presentada sirva para consolidar el estudio de todas las formas de juego y resulte útil a las personas e instituciones interesadas en diseñar experiencias que promuevan la competencia cívica de los potenciales jugadores.

\section{Referencias bibliográficas}

Álvarez-Arregui, E. (2019). Evolución de la Universidad en la Sociedad del Aprendizaje y la Enseñanza. El valor de las competencias en el desarrollo profesional y personal. Aula Abierta, 48, 4, 349-373

Ashtari, D., y de Lange, M. (2019). Playful civic skills: A transdisciplinary approach to analyse participatory civic games. Cities, 89, 70-79. https://doi.org/10.1016/j.cities.2019.01.022

Bogdan, R., y Biklen, S. (2007). Qualitative research for education: An introduction to theory and practice. Boston: Allyn and Bacon.

Bogost, I. (2015). Why gamification is bullshit. En S. Walz \& S. Deterding, S. (Eds.), The Gameful World: Approaches, Issues, Applications, (pp 65-80). Cambridge, MA: MIT Press. https:/ / doi.org/10.7551/mitpress/9788.003.0005

Brookes, S., y Moseley, A. (2012). Authentic contextual games for learning. En N. Whitton \& A. Moseley (Eds), Using Ga- mes to Enhance Learning and Teaching: A Beginner's Guide (pp. 91-107). New York, NY: Routledge.

Caillois, R. (1961). Man, Play, and Games. Chicago, IL: University of Illinois Press.

Comisión Europea. (2007). Competencias clave para el aprendizaje permanente. Un marco de referencia Europeo. Recuperado de http://www.mecd.gob.es/dctm/ministerio/educacion/ mecu/movilidad-europa/competenciasclave.pdf?documen$\mathrm{tId}=0901 \mathrm{e} 72 \mathrm{~b} 80685 \mathrm{fb} 1$

Consejo de Europa. (2000). Conclusiones de la presidencia. Recuperado de http://www.europarl.europa.eu/summits/lis1_ es.htm

Consejo Europeo. (2001). Futuros objetivos precisos de los sistemas educativos y formación (5980/01 EDUC23). Recuperado de https:/ / www.sepe.es/contenidos/personas/formacion/refernet/pdf/05980es1\%28futurosobjprecisos\%29.pdf.

Consejo Europeo. (2010). Conclusiones del Consejo de 12 de mayo de 2009 sobre un marco estratégico para la cooperación europea en el ámbito de la educación y la formación (ET 2020 C119/02). Recuperado de http://e-spacio.uned.es/fez/eserv/bibliuned:reec-2010-16-5230/Documento.pdf

Creswell, J. W. (2011). Educational Research: Planning, Conducting, and Evaluating Quantitative and Qualitative Research). Boston: Pearson.

Del Moral, M. E., y Villalustre, L. (2006). Gameproyect: una estrategia metodológica que favorece el trabajo colaborativo basado en las Webquests. Aula abierta, (87), 123-146.

Deterding, S., Dixon, D., Khaled, R., y Nacke, L. (2011). From game design elements to gamefulness: defining gamification. En A. Lugmayr (Ed.), Proceedings of the 15th international academic MindTrek conference: Envisioning future media environments (pp. 9-15). Tampere: Finlandia: ACM. Recuperado de https: / /dl.acm.org/ citation.cfm?id=2181040

Fuchs, M., Fizek, S., y Ruffino, P. (2014). Rethinking Gamification. Lüneburg, Alemania: Meson Press. https://doi. org/10.14619/001

Fuster, H. (2017). Influencia de las Motivaciones y los Estilos de Juego en Jugadores de MMORPG (Tesis doctoral). Recuperado de https://www.tdx.cat/bitstream/handle/10803/403376/ Tesi_Hector_Fuster.pdf

Gee, J. P. (2014). What Video Games Have to Teach Us About Learning and Literacy. Second Edition. Hampshire, UK: Macmillan. https: / /doi.org/10.1145/950566.950595

George, M., y Apter, A. J. (2004). Gaining insight into patients' beliefs using qualitative research methodologies. Current Opinion in Allergy and Clinical Immunology, 4(3), 185-189.

Hamari, J., Shernoff, D. J., Rowe, E., Coller, B., Asbell-Clarke, J., y Edwards, T. (2016). Challenging games help students learn: An empirical study on engagement, flow and immersion in game-based learning. Computers in Human Behavior, 54, 170179. https: / / doi.org/ 10.1016/j.chb.2015.07.045

Hartas, D. (2015). Educational research and inquiry: Qualitative and quantitative approaches. London, UK: Bloomsbury Academic.

Huizinga, J. (1949). Homo Ludens. London, UK: Taylor \& Francis.

Hunicke, R., Leblanc, M., y Zubek, R. (2004). MDA: A formal approach to game design and game research. En D. Fu y J Orkin (Eds.), Proceedings of the Challenges in Games AI Workshop, 19th National Conference of Artificial Intelligence (pp. 1-5). Menlo Park, CA: AAAI Press

Jenkins, H. (2005). Games, the new lively art. En J. Raessens y J. Goldstein (Eds.), Handbook of Computer Game Studies (pp 175-189). Cambridge, MA: MIT Press

Lazzaro, N. (2004). Why We Play Games: Four Keys to More Emotion Without Story. Presented at Trabajo presentado en Game De- 
velopers Conference. Recuperado de http:/ / xeodesign.com/ xeodesign_whyweplaygames.pdf

Manninen, T. (2003). Interaction forms and communicative actions in multiplayer games. Game Studies, 3(1). Recuperado de http:/ / www.gamestudies.org/0301/manninen/

Marín, V. (2006). Los videojuegos en el ámbito de la familia: Propuesta de trabajo colaborativo. Aula abierta, 87, 71-84.

Martínez-Salgado, C. (2012). El muestreo en investigación cualitativa. Principios básicos y algunas controversias. Revista Ciência \& Saúde Coletiva, 17(3). Recuperado de http: / / www.scielo.br/scielo.php?script=sci_arttext\&pi$\mathrm{d}=\mathrm{S} 1413-81232012000300006$

Mertens, D. M. (2014). Research and evaluation in education and psychology: Integrating diversity with quantitative, qualitative, and mixed methods. Thousand Oaks, CA: Sage.

Miles, M. B., Huberman, A. M., y Saldana, J. (2014). Qualitative data analysis: A method sourcebook. Thousand Oaks, CA: Sage. https:/ / doi.org/10.1080/10572252.2015.975966

Montola, M., Stenros, J., y Waern, A. (2009). Pervasive Games: Theory and Design. Burlington, MA: Kaufmann

Neys, J., y Jansz, J. (2019). Engagement in play, engagement in politics: Playing political video games. En R. Glass, S. Lammes, M. de Lange, J. Raessens \& I. de Vries (Eds.), The Playful Citizen, (pp. 36, 55). Amsterdam, Paises Bajos: Amsterdam University Press. Recuperado de https://www.oapen.org/ download?type $=$ document $\&$ docid $=1004135 \#$ page $=37$

Oceja, J., y González-Fernández, N. (2016). Actors, Elements, and Innovative Interfaces in Game Experiences: CCAE as a Model For Analysing Game Elements. En T. Connolly y L. Boyle (Eds.), European Conference on Games Based Learning (p. 509514). Peasley, Escocia: ACI. Recuperado de https://www. researchgate.net/publication/308678482_Actors_Elements_ and_Innovative_Interfaces_in_Game_Experiences_CCAE_ as_a_Model_For_Analysing_Game_Elements

Oceja, J., y González-Fernandez, N. (2017). Classification of game experiences to promote civic competence in the context of informal learning. En M.Pijec y J. Gründler (Eds.) European Conference on Games Based Learning (p. 480-487). Graz, Austria: ACI. Recuperado de https://search.proquest.com/ openview /6716a7636f98ec36fad90ccb43c19b6a/1?pq-origsite $=$ gscholar\&cbl=396495

Oceja, J., y González-Fernández, N. (2018). Videojuegos y aprendizaje. ¿Por qué la gamificación y los juegos educativos no son suficientes? [Video games and learning. Why gamification and educational games are not enough?] In A. To-
rres-Toukoumidis \& L.M Romero-Rodríguez (Eds.) Gamificación en Iberoamérica. Experiencias desde la Comunicación y la Educación. Quito: Abya-Yala

Oceja, J., y González-Fernández, N. (2020). University students and video games: Perceptions, use, and preferences according to gender. Education policy analysis archives, 28, 66. https://doi.org/10.14507/epaa.28.4181

Raessens, J. (2006). Playful identities, or the ludification of culture. Games and Culture, 1(1), 52-57. https://doi. org /10.1177/1555412005281779

Robinson, W. S. (2000). The logical structure of analytic induction. En R. Gomm, M. Hammersley y P. Foster (Eds.), Case Study Method (pp. 187-195). London, UK: Sage.

Schell, J. (2014). The Art of Game Design: A Book of Lenses. Boca Ratón, FL: CRC Press. https:/ / doi.org/ 10.1201/9780080919171

Schettini, P. (2015). Análisis de datos cualitativos en la investigación social. Buenos Aires, Argentina: Editorial de la Universidad de La Plata.

Shaffer, D. W., Squire, K. R., Halverson, R., y Gee, J. P. (2005). Video games and the future of learning. Phi Delta Kappan, 87(2), 105-111.

Spradley, J. P. (2016). The ethnographic interview. Long Grove, IL: Waveland Press.

Strauss, A., y Corbin, J. M. (1997). Grounded theory in practice. Thousand Oaks, CA: Sage.

Tesch, R. (2013). Qualitative Types: Analysis Typ. Oxon, UK: Routledge.

Tschang, F. T. (2007). Balancing the tensions between rationalization and creativity in the video games industry. Organization Science, 18(6), 989-1005.

Vorderer, P., Klimmt, C., y Ritterfeld, U. (2004). Enjoyment: At the Heart of Media Entertainment. Communication Theory, 14(4), 388-408. https://doi.org/10.1111/j.1468-2885.2004.tb00321.x

Zaffiro, G., Melissa, B., Brynskov, M., y Smyth, M. (2015). Designing digital urban interactions industry landscape and market analysis. En P. Kommers y P. Isaias (Eds.), Proceedings of the 13th International Conference e-Society 2015 (pp. 249-254). Madeira, Portugal: IADIS

Zagalo, N., Carvalho, A. A., y Araújo, I. (2016). Elementos do design de videojogos que fomentam o interesse dos jogadores. Revista ESC-Educação, Sociedade E Culturas, 48, 169-190. https://doi.org/ 10.1016/j.chb.2004.11.011 